\title{
A Monitoring Strategy for Industry 4.0: Master Italy s.r.l Case Study
}

\author{
Concetta Semeraro ${ }^{1,2,3}$, Hervé Panetto ${ }^{2}$, Mario Lezoche², Michele Dassisti ${ }^{1}$, \\ Stefano Cafagna ${ }^{3}$ \\ ${ }^{1}$ Department of Mechanics, Management \& Mathematics (DMMM), Polytechnic of Bari, Bari, Italy \\ ${ }^{2}$ Université de Lorraine, CNRS, CRAN, Nancy, France \\ ${ }^{3}$ Master Italy s.r.l, Conversano, Bari, Italy
}

Manufacturing enterprises are presently facing with an array of industry 4.0 (I4.0) challenges. "Digital requirements" need to be really assessed by an accurate analysis and deep understanding of the operational and technological criticalities in the manufacturing operations.

It is required to structure a monitoring strategy for industry 4.0 that contains:

1. The definition of the measuring parameters.

2. The application of the sensor.

3. The execution of the measurement.

The goal in fact is to present and to analyse the monitoring strategy adopted in a design for the digital transformation of a real Italian SME company: Master Italy s.r.l.

Master Italy s.r.l. is a SME Italian company producing small accessories for civil window frames, is here considered to implement a monitoring strategy.

The monitoring strategy is a hybrid approach between the life cycle analysis (LCA) and the exergetic analysis (EA) based on the evaluation of the mass balance (Fig.1.) and the energy balance (Fig.2.).

LCA is an analytical tool used to quantify and to interpret the flows to-and-from the environment through the whole life cycle of a product, process or service. LCA appreciates quantities of elements flowing in the processes (say, energy, materials, etc.) but it depends on standard databases.

The exergetic analysis is a thermodynamic method [1] that permits to:

- Evaluate the quality of energy usage.

- Identify and quantify the energy inefficiency of the process.

Life cycle analysis (LCA) enables to identify the critical manufacturing process and the critical product of the company in terms of resource consumption and pollutions (green line). The critical process and the critical product in analysis are respectively the die casting aluminium and the steel corner (Fig. 1 and Fig.2).

The exergetic analysis allows to:

1. Split the manufacturing process in different sub-systems.

2. Identify the critical sub-system evaluating the contribute of the exergy loss ( $\left.E_{x l o s s}\right)$.

3. Define the critical parameters to control.

Die casting is a metal casting process that is characterized by forcing molten metal under high pressure into a mould cavity. The injection cycle of die casting aluminium process is composed by four different phases:

1. Melting: the aluminium enters at the solid state and exits at the molten state.

2. Injection: the molten aluminium is transferred, thought a plunger, into a chamber where it is injected into the mould.

3. Moulding: the molten aluminium solidifies in the mould cavity.

4. Extraction: an ejection mechanism pushes the casting out of the mould cavity.

The optimization criterion of the monitoring strategy is to minimize the term $E_{x l o s s}$, since the exergy loss is proportional to the generated entropy and this one is responsible for the less-than-theoretical efficiency of the system. The application of the exergetic analysis shows that the moulding phase (Subsystem 3) is the critical subsystem because the exergy loss is highest than other subsystems (Fig.1 and Fig.2). The first goal of the monitoring strategy, for Industry 4.0 implementations, is to select and to define where, what and why to sensorize (red rectangle). 


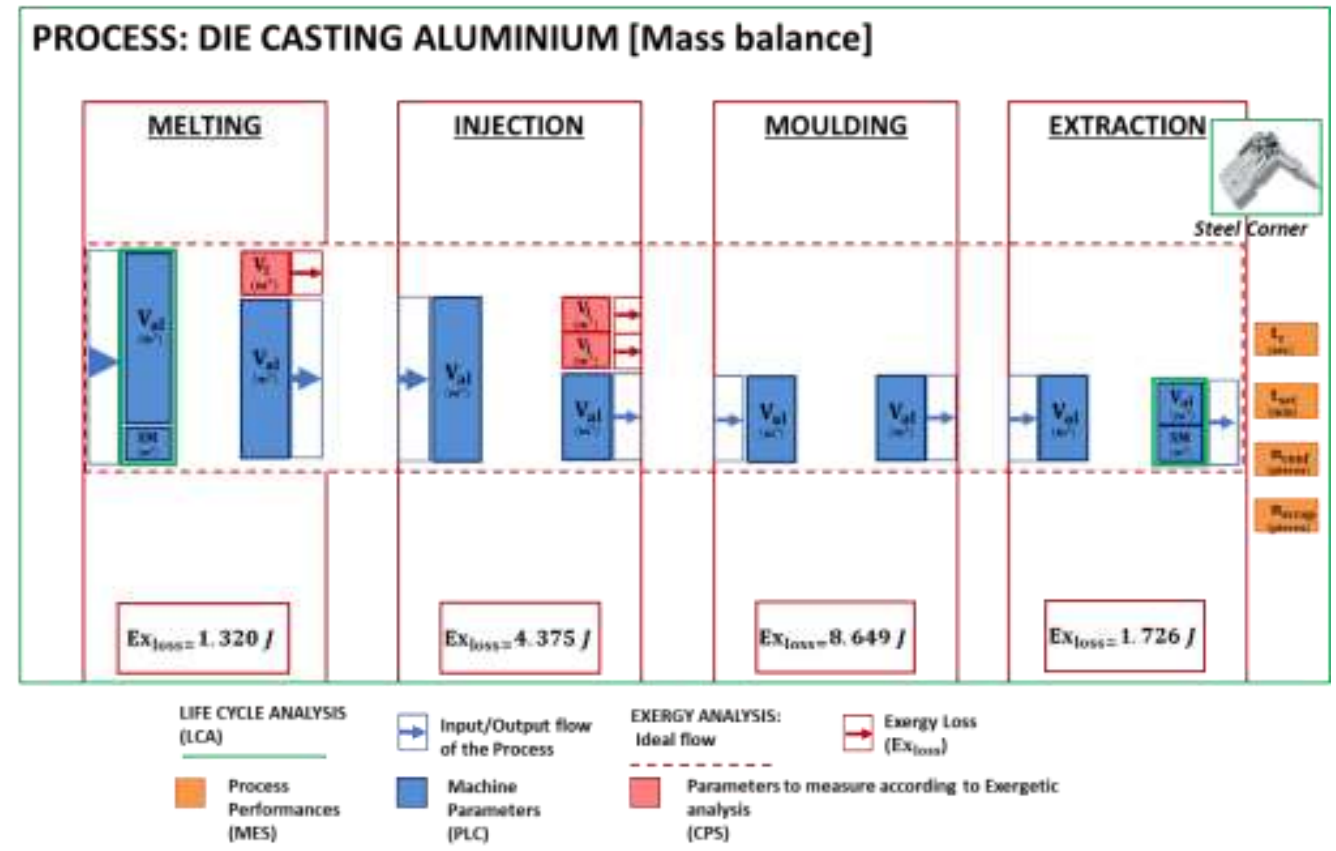

Fig.1. Mass Balance

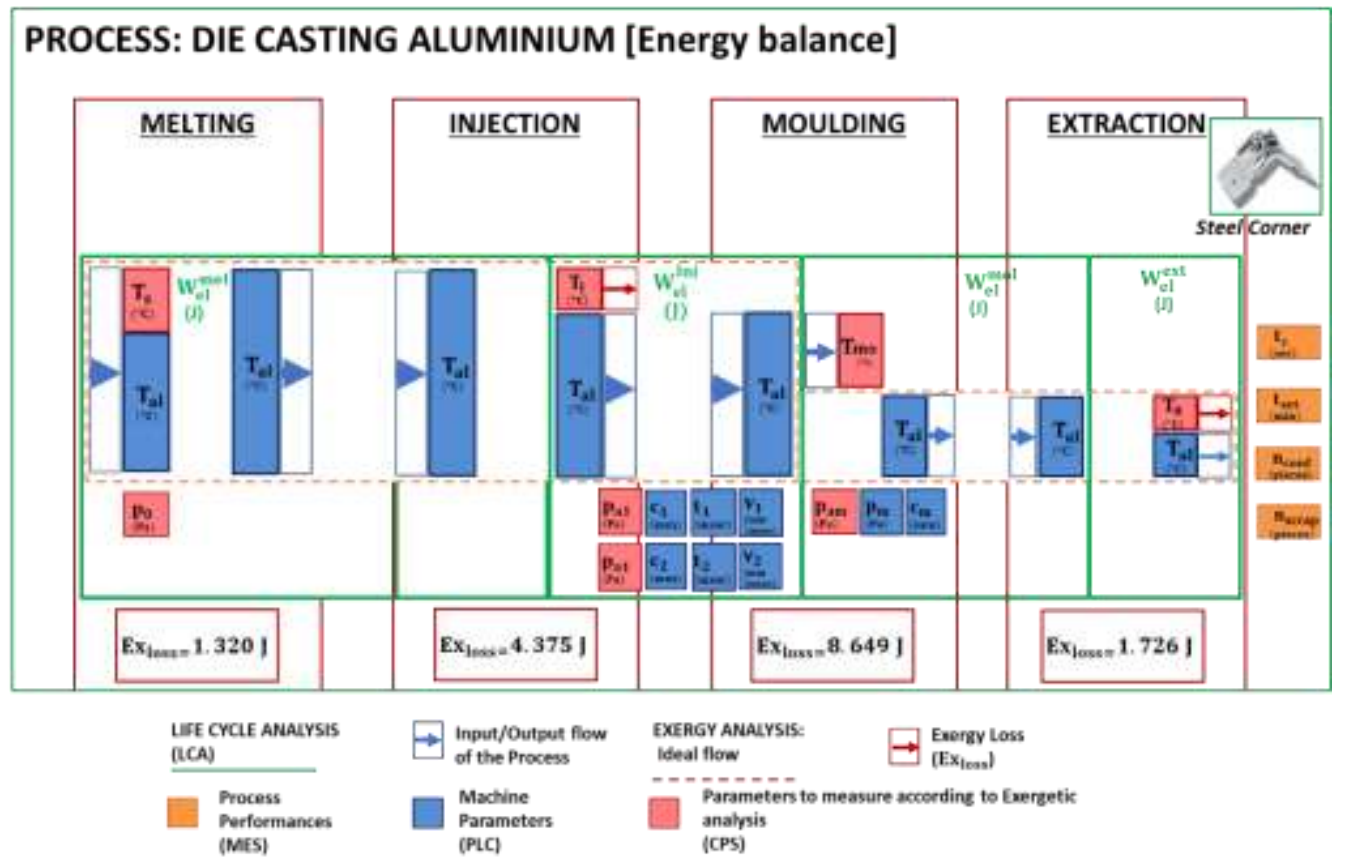

Fig.2. Energy Balance

The term exergy loss depends on the variation between input and output of material exergy $\left(E_{\mathrm{x}, \mathrm{M}}\right)$, work exergy $\left(E_{\mathrm{x}, \mathrm{W}}\right)$, and heat exergy $\left(\mathrm{E}_{\mathrm{x}, \mathrm{Q}}\right)$. Reducing the exergy loss means to predict process behaviour: it is thus necessary to identify and classify the parameters to monitor within the main, derived and noncontrollable ones.

Data is an important element for monitoring and modelling complex systems. Data contains information and it is useful for modelling, simulation, optimization, and prediction. With the developments of New Information Technologies, there is the increase of the volume, richness, and fidelity of data from the physical and virtual spaces. The monitoring strategy, in this stage, aims to integrate and to fuse the data from the different sources in order to obtain and to extract more accurate and useful information from data (Fig.3).

In this way, operational, technological and environmental data from the physical space can be used to simulate results and to predictive states in the virtual space. 
It means that it is necessary to access to realistic models of the current state of the process. These models are typically called digital twin (Fig.3) [2].

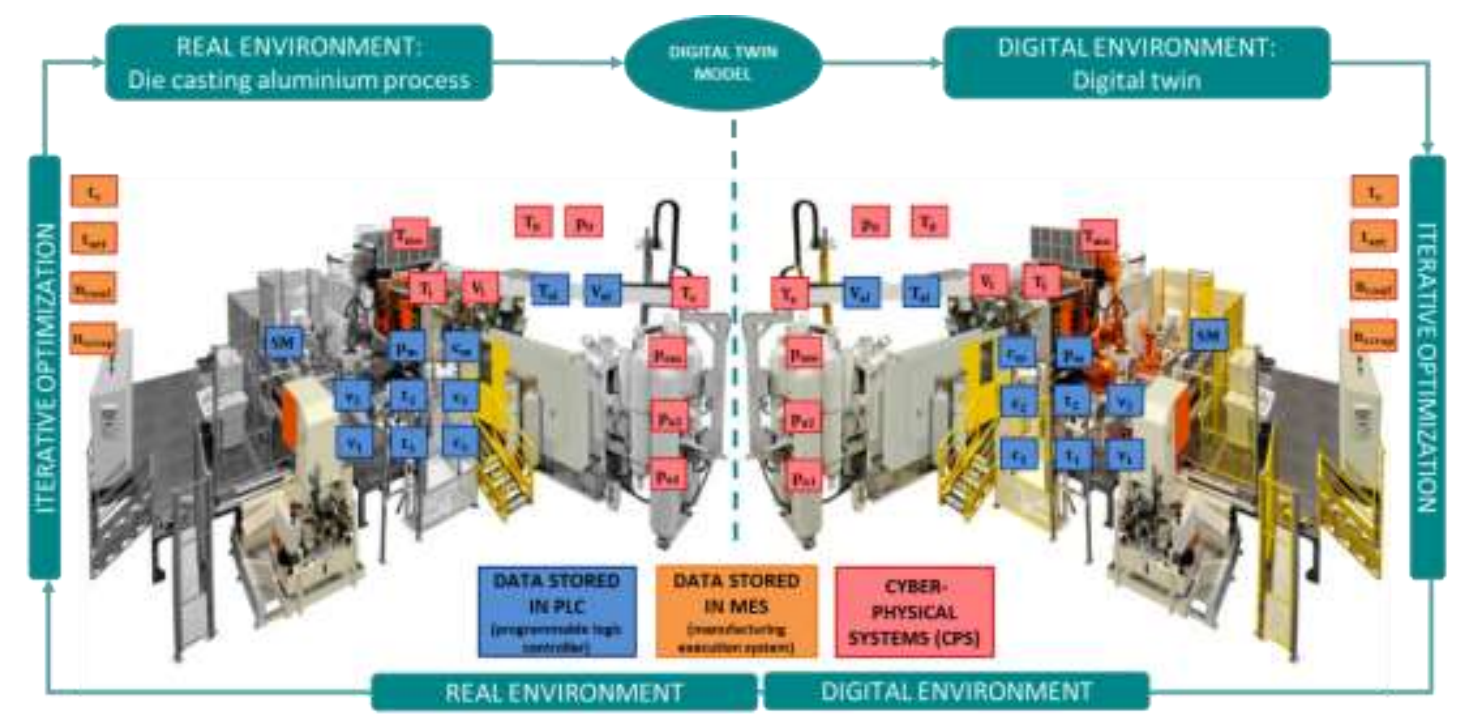

Fig.3. The Digital Twin

The digital twin is defined as a set of models that emulate the physical properties, the behaviours and the constrains of the manufacturing process and it gets real time data to update itself along the product lifecycle [3]. The digital twin is the digital mirror of the physical process [4].

The architecture proposed is composed of five layers [5] [6]:

1. Physical layer which includes production factors: human, equipment, materials and environment.

2. Data layer includes production data, tooling data, equipment data, material data, quality data, cost data, human data, environmental data etc, which are the base of the cyber layer.

3. Network layer: through which the cyber and physical layer can communicate real time. It is critical to build a connection between the physical and virtual entities for data exchange.

4. Cyber layer which is the digital model of the physical layer. The cyber layer compares simulated results with known information, represented by mathematical or physical equations. The digital model is based on a set of different models to represent the structure, the behaviour and the interactions of a physical system to be monitored or predicted. SysML (Systems Modelling Language) is the modelling language used for building physical, behaviour and parametric models. SysML provides nine interrelated types of diagrams to describe the function, the structure, the behaviour and the system requirements. It supports the specification, analysis, and verification of systems' models. The subsystems are modelled as blocks and the parameters as value properties using the block definition diagram (bdd). The behaviour is modelled as a set of actions to describe how the inputs are transformed into outputs. In particular, the state diagram (stm) models the behaviour defining states and events of an object during its lifetime. It simulates how the states change based on internal or external events. The parametric diagram (par) models the exergetic equations in terms of constraints. Constraints represent physical laws or mathematical and logical operators or decisions that evaluate input parameters to return a result.

5. Application and user interface layer which provides service to support the management and control of physical layer through the cyber layer. It tries to make the PL work as expected through real-time regulation, and sustains high fidelity of the CL through model parameters calibration. 


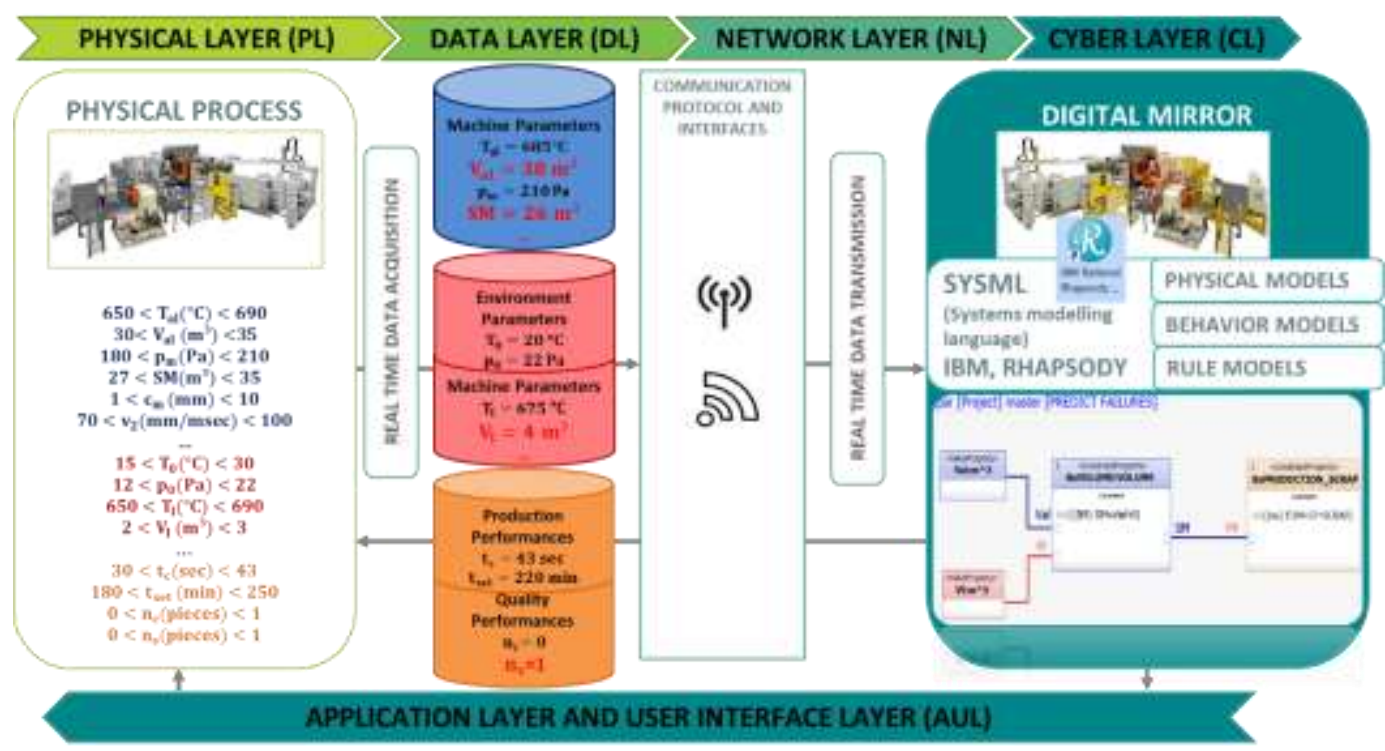

Fig.4. The Architecture for Monitoring Strategy

The proposed architecture can be applied on manufacturing process for [7]:

- Geometry assurance - The digital twin accelerates the correct design product developing.

- Remote monitoring - The digital twin allows remote visibility of the operations of large interconnected systems such as manufacturing systems, which allows virtual monitoring systems and validation of the current status of production systems (i.e. energy monitoring and fault monitoring).

- Predictive analytics - Prediction of the future state of the digital twin can be used to predict errors and problems in manufacturing facilities before they occur, therefore preventing downtime, failures and more.

- Simulating future behaviour - The digital twin can be used by virtually simulating manufacturing processes to plan, reconfiguration of processes and the system in response to external changes.

- Optimization and validation - Validate and optimize the system's operation using simulation and real-time sensor feedback.

\section{References}

[1] B. R. Bakshi, T. G. Gutowski, and D. P. Sekulic, Thermodynamics and the Destruction of Resources. Cambridge University Press, 2011.

[2] R. Rosen, G. von Wichert, G. Lo, and K. D. Bettenhausen, "About the importance of autonomy and digital twins for the future of manufacturing," IFAC-Pap., vol. 48, no. 3, pp. 567-572, 2015.

[3] M. Grieves and J. Vickers, "Digital twin: Mitigating unpredictable, undesirable emergent behavior in complex systems," in Transdisciplinary Perspectives on Complex Systems, Springer, 2017, pp. 85-113.

[4] E. Glaessgen and D. Stargel, "The digital twin paradigm for future NASA and US Air Force vehicles," in 53rd AIAA/ASME/ASCE/AHS/ASC Structures, Structural Dynamics and Materials Conference 20th AIAA/ASME/AHS Adaptive Structures Conference 14th AIAA, 2012, p. 1818.

[5] F. Tao and M. Zhang, "Digital twin shop-floor: a new shop-floor paradigm towards smart manufacturing," Ieee Access, vol. 5, pp. 20418-20427, 2017.

[6] K. Ponomarev, N. Kudryashov, N. Popelnukha, and V. Potekhin, "Main principals and issues of digital twin development for complex technological processes," presented at the Annals of DAAAM and Proceedings of the International DAAAM Symposium, 2017, pp. 523-528.

[7] J. Lee, E. Lapira, S. Yang, and A. Kao, "Predictive manufacturing system-Trends of next-generation production systems," IFAC Proc. Vol., vol. 46, no. 7, pp. 150-156, 2013. 